\title{
Corrigendum: Safety of Semaglutide
}

\author{
Mark M. Smits and Daniël H. Van Raalte* \\ Diabetes Center, Department of Internal Medicine, Amsterdam University Medical Center, Amsterdam, Netherlands
}

\section{OPEN ACCESS}

Edited by:

Francesco Giorgino,

University of Bari Aldo Moro, Italy

Reviewed by:

Stephen Bain,

Swansea University, United Kingdom

*Correspondence:

Daniël H. Van Raalte

d.vanraalte@amsterdamumc.nl

Specialty section: This article was submitted to

Clinical Diabetes, a section of the journal

Frontiers in Endocrinology

Received: 30 September 2021

Accepted: 15 October 2021

Published: 10 November 2021

Citation:

Smits MM and Van Raalte DH (2021) Corrigendum: Safety of Semaglutide.

Front. Endocrinol. 12:786732. doi: 10.3389/fendo.2021.786732
Keywords: glucagon-like peptide-1 receptor agonist (GLP-1RA), oral, subcutaneous, semaglutide, type 2 diabetes, safety

\section{A Corrigendum on}

\section{Safety of Semaglutide}

By Smits MM and Van Raalte DH (2021) Front. Endocrinol. 12:645563. doi: 10.3389/fendo.2021.645563

In the original article, there was a mistake in Table 2. Adverse effects and safety risks in phase 3 trials as published. Three values in the row for SUSTAIN 6, s.c semaglutide 1 mg were accidentally transposed to the incorrect column. The corrected Table 2 appears below.

The authors apologize for this error and state that this does not change the scientific conclusions of the article in any way. The original article has been updated.

Publisher's Note: All claims expressed in this article are solely those of the authors and do not necessarily represent those of their affiliated organizations, or those of the publisher, the editors and the reviewers. Any product that may be evaluated in this article, or claim that may be made by its manufacturer, is not guaranteed or endorsed by the publisher.

Copyright (C) 2021 Smits and Van Raalte. This is an open-access article distributed under the terms of the Creative Commons Attribution License (CC BY). The use, distribution or reproduction in other forums is permitted, provided the original author(s) and the copyright owner(s) are credited and that the original publication in this journal is cited, in accordance with accepted academic practice. No use, distribution or reproduction is permitted which does not comply with these terms. 
TABLE 2 | Adverse effects and safety risks in phase 3 trials (10-32).

Treatment arms

Incidence of $\mathrm{AE}, \mathrm{n}(\%)$

$\%$ of patients with $A E$

leading to trial product

discontinuation

Any Severe or confirmed

\section{Pancreas}

Gallbladder Thyroid Diabetic

Any Gastrointestinal,

Acute

ymptomatic hypoglycemic episode*

cancer

\section{PIONEER 1}

5 (2.9)

$2(1.1)$

(53.1)

Placebo

99

PIONEER 2

Oral semaglutide $14 \mathrm{mg} \quad 289$

(70.5)

Empagliflozin $25 \mathrm{mg} \quad 283$

PIONEER 3

Oral semaglutide $3 \mathrm{mg} \quad 370$

(78.2)

Oral semaglutide $14 \mathrm{mg} \quad 370$

PIONEER 4

$\begin{array}{ll} & (80) \\ \text { Liraglutide } 1.8 \mathrm{mg} \text { (s.c.) } & 211\end{array}$

PIONEER 5

Placebo

\section{PIONEER 6}

Oral semaglutide $14 \mathrm{mg} \quad \mathrm{NR}$

Placebo

NR

PIONEER 7

Oral semaglutide $\quad 197 \quad 14(5.5)$

(flexible 3, 7 or $14 \mathrm{mg}$ )

Sitagliptin $100 \mathrm{mg}$
Oral semaglutide $7 \mathrm{mg} \quad(57.7)$

(56.6)

Oral semaglutide $7 \mathrm{mg} \quad(79.4)$

Sitagliptin $100 \mathrm{mg} \quad 388$

Oral semaglutide $14 \mathrm{mg} \quad 229$

$\begin{array}{lr} & (74) \\ \text { Placebo } & 95(67)\end{array}$

Oral semaglutide $14 \mathrm{mg} \quad 122$

\section{$1(0.6)$}

1 (0.6)

$7(1.7)$

$8(2.0)$

$23(4.9)$

$24(5.2)$

$36(7.7)$

$39(8.4)$

$$
2(1)
$$

7 (2)

3 (2)

$9(6)$

3 (2)

NR

NR

14 (5.6)

$\begin{array}{cccc}14(8.0) & 5(2.9) & 15(8.6) & 0 \\ 9(5.1) & 8(4.6) & 9(5.1) & 0 \\ 28 & 12(6.9) & 9(5.1) & 0 \\ (16.0) & & & \\ 10(5.6) & 4(2.2) & 4(2.2) & 0\end{array}$

$81 \quad 30(7.3) \quad 38(9.3) \quad 1(0.2)$

$10(2.4) \quad 7(1.7) \quad 13(3.2) \quad 1(0.2)$

$10(2.4) \quad 7(1.7) \quad 13(3.2) \quad 1(0.2)$

$34(7.3) \quad 13(2.8) \quad 45(9.7) \quad 1(0.2)$

$62 \quad 28(6.0) \quad 53(11.4) \quad 1(0.2)$

$(13.4)$
70 $42(9.0) \quad 57(12.3) \quad 1(0.2)$

$\begin{array}{ccccc}70 & 42(9.0) & 57(12.3) & 1(0.2) & 1(0.2) \\ (15.1) & & & & \\ 32(6.9) & 19(4.1) & 37(7.9) & 1(0.2) & 1(0.2)\end{array}$

$56(20) \quad 25(9) \quad 43(15) \quad 0$

$51(18) \quad 13(5) \quad 31(11) \quad 1(0.4)$

$5(4) \quad 3(2) \quad 11(8) \quad 1(0.7)$

$31(19) \quad 19(12) \quad 17(10)$

$12(7) \quad 6(4) \quad 6(4)$

$\begin{array}{llll}N R & N R \quad N R & 1(0.1)\end{array}$

$53(21) \quad 14(6) \quad 22(9) \quad 0$

$6(2) \quad 3(1) \quad 8(3)$
$\begin{array}{lll}N R & N R \quad N R \quad 3(0.2)\end{array}$

retinopathy

Injury (\%)

$\%$

NR

0

$1(0.6 \%)$

$4(2.3)$

75

$\begin{array}{lllll}N R & 0 & 6(3.4 \%) & 0 & 7(4.0)\end{array}$

$\begin{array}{lllll}N R & 0 & 2(1.1 \%) & 1(0.6) & 13(7.4)\end{array}$

$\begin{array}{llllll}\mathrm{NR} & 0 & 3(1.7 \%) & 1(0.6) & 4(2.2) & 25\end{array}$

$\begin{array}{lllllcl}0 & N R & 0 & 14(3.4) & 2(0.5) & 44 & 75\end{array}$

0

NR

$0 \quad 5(1.2 \%) \quad 1(0.2) \quad 18(4.4)$

$\begin{array}{lllllll}0 & N R & 0 & 27(5.8) & 3(0.6) & 26(5.6) & 42\end{array}$

$\begin{array}{llllll}0 & N R & 0 & 24(5.2) & 2(0.4) & 27(5.8)\end{array}$

$\begin{array}{llllll}1(0.2) & N R & 0 & 16(3.4) & 5(1.1) & 54\end{array}$

$1(0.2) \quad \mathrm{NR}$

$\begin{array}{llll}0 & 27(5.8) & 3(0.6) & 24(5.2)\end{array}$

59

$\begin{array}{ll} & 172 \\ & (69)\end{array}$

$\begin{array}{ll}72 & 14(5.6)\end{array}$




\begin{tabular}{|c|c|c|c|c|c|c|c|c|c|c|c|c|c|}
\hline \multirow[t]{3}{*}{ Treatment arms } & \multicolumn{11}{|c|}{ Incidence of AE, n (\%) } & \multicolumn{2}{|c|}{$\begin{array}{l}\% \text { of patients with } \mathrm{AE} \\
\text { leading to trial product } \\
\text { discontinuation }\end{array}$} \\
\hline & \multirow[t]{2}{*}{ Any } & \multirow{2}{*}{$\begin{array}{c}\text { Severe or confirmed } \\
\text { symptomatic hypoglycemic } \\
\text { episode* }\end{array}$} & \multicolumn{3}{|c|}{ Gastrointestinal } & \multicolumn{2}{|c|}{ Pancreas } & \multirow[t]{2}{*}{ Gallbladder } & \multirow[t]{2}{*}{ Thyroid } & \multirow{2}{*}{$\begin{array}{l}\text { Diabetic } \\
\text { retinopathy }\end{array}$} & \multirow{2}{*}{$\begin{array}{l}\text { Acute } \\
\text { kidney } \\
\text { Injury }\end{array}$} & \multirow{2}{*}{$\begin{array}{l}\text { Any } \\
\text { AE, n } \\
(\%)\end{array}$} & \multirow{2}{*}{$\begin{array}{c}\text { Gastrointestinal, } \\
\%\end{array}$} \\
\hline & & & Nausea & Vomiting & Diarrhea & Pancreatitis & $\begin{array}{l}\text { Pancreatic } \\
\text { cancer }\end{array}$ & & & & & & \\
\hline \multicolumn{14}{|l|}{ PIONEER 8} \\
\hline Oral semaglutide $3 \mathrm{mg}$ & $\begin{array}{c}137 \\
(74.5)\end{array}$ & $52(28.3)$ & $\begin{array}{c}21 \\
(11.4)\end{array}$ & $11(6.0)$ & $16(8.7)$ & 0 & 0 & NR & 0 & $7(3.8)$ & $2(1.1)$ & $13(7.1)$ & 69 \\
\hline Oral semaglutide $7 \mathrm{mg}$ & $\begin{array}{c}142 \\
(78.5)\end{array}$ & 47 (26.0) & $\begin{array}{c}30 \\
(16.6)\end{array}$ & $14(7.7)$ & 22 (12.2) & 0 & 0 & NR & 0 & $8(4.4)$ & $1(0.6)$ & $16(8.8)$ & 75 \\
\hline Oral semaglutide 14 mg & $\begin{array}{c}151 \\
(83.4)\end{array}$ & $48(26.5)$ & $\begin{array}{c}42 \\
(23.2)\end{array}$ & $18(9.9)$ & 27 (14.9) & 0 & 0 & NR & 0 & $9(5.0)$ & 0 & $\begin{array}{c}24 \\
(13.3)\end{array}$ & 79 \\
\hline Placebo & $\begin{array}{c}139 \\
(75.5)\end{array}$ & $54(29.3)$ & $13(7.1)$ & $7(3.8)$ & $11(6.0)$ & 0 & 0 & $N R$ & 0 & $8(4.3)$ & 0 & $5(2.7)$ & 20 \\
\hline \multicolumn{14}{|l|}{ PIONEER 9} \\
\hline Oral semaglutide $3 \mathrm{mg}$ & $37(76)$ & 0 & $2(4)$ & NR & $4(8)$ & 0 & 0 & NR & 0 & 0 & 0 & $1(2)$ & 100 \\
\hline Oral semaglutide $7 \mathrm{mg}$ & $37(76)$ & 0 & $5(10)$ & NR & $1(2)$ & 0 & 0 & NR & 1 & $1(2.0)$ & 0 & $1(2)$ & 100 \\
\hline Oral semaglutide 14 mg & $34(71)$ & 0 & $4(8)$ & NR & $3(6)$ & 0 & 0 & NR & 0 & $1(2.1)$ & 0 & $2(4)$ & 100 \\
\hline Liraglutide 0.9 mg (s.c.) & $32(67)$ & $2(4.2)$ & 0 & NR & $2(4)$ & 0 & 0 & NR & 0 & 0 & 0 & 0 & 0 \\
\hline \multicolumn{12}{|l|}{ PIONEER 10} & 0 & 0 \\
\hline Oral semaglutide $3 \mathrm{mg}$ & $\begin{array}{l}101 \\
(77)\end{array}$ & $3(2)$ & $7(5)$ & $3(2)$ & $2(2)$ & 0 & 0 & $2(2)$ & 0 & $9(7)$ & 0 & $4(3)$ & 50 \\
\hline Oral semaglutide $7 \mathrm{mg}$ & $\begin{array}{l}106 \\
(80)\end{array}$ & $3(2)$ & $11(8)$ & $6(5)$ & $2(2)$ & 0 & 0 & $1(1)$ & 0 & $12(9)$ & 0 & $8(6)$ & 50 \\
\hline Oral semaglutide 14 mg & $\begin{array}{l}111 \\
(85)\end{array}$ & $4(3)$ & $12(9)$ & $9(7)$ & $10(8)$ & 0 & 0 & 0 & 0 & $7(5)$ & 0 & $8(6)$ & 63 \\
\hline Dulaglutide 0.75 mg (s.c.) & $53(82)$ & 0 & $6(9)$ & $1(2)$ & $4(6)$ & 0 & 0 & $1(2)$ & 0 & $3(5)$ & 0 & $2(3)$ & 50 \\
\hline \multicolumn{14}{|l|}{ SUSTAIN 1} \\
\hline S.c. semaglutide $0.5 \mathrm{mg}$ & $82(64)$ & 0 & $26(20)$ & $5(4)$ & $16(13)$ & 0 & 0 & $3(2)$ & 0 & NR & 0 & $8(6)$ & 63 \\
\hline S.c. semaglutide $1 \mathrm{mg}$ & $73(56)$ & 0 & $31(24)$ & $9(7)$ & $14(11)$ & 0 & 0 & $1(<1)$ & 0 & NR & 0 & $7(5)$ & 57 \\
\hline \multicolumn{14}{|l|}{ SUSTAIN 2} \\
\hline S.c. semaglutide $0.5 \mathrm{mg}$ & $\begin{array}{l}306 \\
(75)\end{array}$ & $7(2)$ & $73(18)$ & $33(8)$ & $54(13)$ & $3(1 \%)$ & $N R$ & $1(<1)$ & 0 & $1(<1)$ & $N R$ & $33(8)$ & 82 \\
\hline S.c. semaglutide $1 \mathrm{mg}$ & $\begin{array}{l}292 \\
(71)\end{array}$ & $2(<1)$ & $72(18)$ & $41(10)$ & $53(13)$ & $1(<1)$ & NR & $7(2)$ & 1 & 0 & NR & $39(10)$ & 79 \\
\hline Sitagliptin 100 & $\begin{array}{l}292 \\
(72)\end{array}$ & $5(1)$ & $30(7)$ & $11(3)$ & $29(7)$ & 0 & $N R$ & $6(1)$ & 0 & $3(1)$ & $N R$ & $12(3)$ & 25 \\
\hline \multicolumn{14}{|l|}{ SUSTAIN 3} \\
\hline S.c. semaglutide $1 \mathrm{mg}$ & $\begin{array}{l}303 \\
(75)\end{array}$ & $33(8.2)$ & $\begin{array}{c}90 \\
(22.3)\end{array}$ & $29(7.2)$ & $46(11.4)$ & $2(<1)$ & NR & $6(1 \%)$ & NR & $N R$ & $N R$ & $38(9.4)$ & $N R$ \\
\hline Exenatide ER 2.0 mg & $\begin{array}{l}309 \\
(76.3)\end{array}$ & $33(8.1)$ & $\begin{array}{c}48 \\
(11.9)\end{array}$ & $25(6.2)$ & $34(8.4)$ & $3(<1)$ & NR & $2(<1)$ & NR & $N R$ & $N R$ & $29(7.2)$ & $N R$ \\
\hline \multicolumn{14}{|l|}{ SUSTAIN 4} \\
\hline S.c. semaglutide $0.5 \mathrm{mg}$ & $\begin{array}{l}253 \\
(70)\end{array}$ & $16(4)$ & $77(21)$ & $24(7)$ & $59(16)$ & $2(1)$ & $1(<1)$ & $1(<1)$ & NR & $1(<1)$ & NR & $20(6)$ & 55 \\
\hline
\end{tabular}




\section{Any Severe or confirmed} symptomatic hypoglycemic episode*

S.c. semaglutide $1 \mathrm{mg}$

Insulin glargine

SUSTAIN 5

S.c. semaglutide $0.5 \mathrm{mg}$

S.c. semaglutide $1 \mathrm{mg} \quad(68.9)$
84

Placebo

$20(6)$

38 (11)

$11(8.3)$

$14(10.7)$

$7(5.3)$

\section{SUSTAIN 6}

$0.5 \mathrm{mg} \quad 740$

(89.6)

S.c. semaglutide $1 \mathrm{mg} \quad 732$
$\quad(89.1)$

Placebo 1484

\section{SUSTAIN 7}

S.c. semaglutide $0.5 \mathrm{mg} \quad 204$

$\begin{array}{ll} & (68) \\ \text { S.c. semaglutide } 1 \mathrm{mg} & 207\end{array}$

$\begin{array}{ll} & (69) \\ \text { Dulaglutide } 0.75 \mathrm{mg} & 186\end{array}$

(s.c.) (62)

Dulaglutide $1.5 \mathrm{mg}$ (s.c.) $\quad 221$

\section{SUSTAIN 8}

S.c. semaglutide $1 \mathrm{mg}$

Canagliflozin $300 \mathrm{mg}$

298

$(76)$
283

(72)

SUSTAIN 9

S.c. semaglutide $1 \mathrm{mg} \quad 104$

$\begin{array}{cc} & (69.3) \\ \text { Placebo } & 91\end{array}$

SUSTAIN 10

S.c. semaglutide $1 \mathrm{mg} \quad 204$

Liraglutide $1.2 \mathrm{mg}$ (s.c.)

(66.2)

\section{1 (23.1)}

$178(21.7)$

$350(21.2)$

$2(1)$

5 (2)

$3(1)$

5 (2)

53 (14)

$32(8)$

17 (11.3)

$3(2.0)$

$5(1.7)$

7 (2.4)
$(2.4)$

Incidence of AE, n (\%)

discontinuation

Pancreas

Gastrointestinal Nausea Vomiting Diarrhea Pancreatitis Pancreatic

cancer

80 (22) $37(10) \quad 69(19) \quad 0 \quad 0$

$13(4) \quad 11(3) \quad 16(4)$

$\begin{array}{cccc}15 & 8(6.1) & 6(4.5) & 0 \\ (11.4) & & & \\ 22 & 15(11.5) & 9(6.9) & 0 \\ (16.8) & & & \\ 6(4.5) & 4(3.0) & 2(1.5) & 0\end{array}$

143
$(17.3)$

$(17.3)$
180
$(21.9)$

$(21.9)$
129

129
$(7.8)$

$68(23)$

$63(21)$

39 (13) $12(4) \quad 23(8)$

$60(20) \quad 29(10) \quad 53(18)$

$89(23) \quad 50(13) \quad 60(15)$

$26(7) \quad 9(2) \quad 37(9)$

$29 \quad 14(9.3) \quad 17(11.3)$

$(19.3)$
$5(3.3) \quad 3(2.0) \quad 9(6.0)$

$(21.8)$
45

(15.7)

0

0

(1.7) $\quad 15(1.8) \quad 6(0.7)$$$
(0.3) \quad 7(0.4) \quad 12(0.7)
$$$$
4(0.2)
$$

0

NR

NR

Gallbladder Thyroid Diabetic

Acute retinopathy kidney kidney
Injury

Any Gastrointestinal,

AE, $n$

(\%)

$\%$

NR 27

2(1) NR

0

NR $\quad 1(<1)$

NR $4(1)$

$\begin{array}{llll}0 & 3(2.3) & 0 & \text { (3.0) }\end{array}$

$\begin{array}{lll}N R & 6(4.5) \quad N R\end{array}$

$\begin{array}{llll}0 & 1(0.8) & 0 & (0.8)\end{array}$

NR $8(6.1)$

NR

O

NR $1(0.8)$

NR

$0 \quad 25(3)$

0

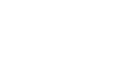

\section{$42(5.1) \quad 95$}

95

$$
49
$$$$
65
$$

$39(2.3) \quad 0$

$29(1.8) \quad 34(4.1) \quad \begin{gathered}(14.5) \\ 110\end{gathered}$

110
$(6.7)$

$\begin{array}{llllll}2(1) & 1(<1) & 2(1) & \mathrm{NR} & 24(8) & 67\end{array}$

$4(1)$

$\begin{array}{llllll}4(1) & 0 & 2(1) & N R & 14(5) & 43\end{array}$

2 (1)

NR $29(10)$

62

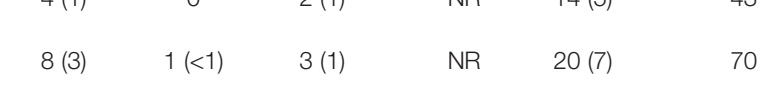

NR

$N R$

$9(2)$

$4(1)$

$38(10)$

NR

NR

$15(4)$

$020(5)$

20

20

$\begin{array}{llllll}\mathrm{NR} & \mathrm{NR} & 3(2.0) & 1(0.7) & 13(8.7) & 77\end{array}$

$\begin{array}{llllll}\mathrm{NR} & \mathrm{NR} & 8(5.3) & 0 & 3(2.0) & 0\end{array}$

$\begin{array}{lllllcl}\mathrm{NR} & \mathrm{NR} & \mathrm{NR} & 3(1.0) & \mathrm{NR} & 33 & 67 \\ \mathrm{NR} & \mathrm{NR} & \mathrm{NR} & 4(1.4) & \mathrm{NR} & \begin{array}{c}(11.4) \\ 19(6.6)\end{array} & 58\end{array}$




\begin{tabular}{|c|c|c|c|c|c|c|c|c|c|c|c|c|}
\hline \multirow[t]{2}{*}{ Any } & \multirow{2}{*}{$\begin{array}{c}\text { Severe or confirmed } \\
\text { symptomatic hypoglycemic } \\
\text { episode }^{*}\end{array}$} & \multicolumn{3}{|c|}{ Gastrointestinal } & \multicolumn{2}{|c|}{ Pancreas } & \multirow[t]{2}{*}{ Gallbladder } & \multirow{2}{*}{ Thyroid } & \multirow{2}{*}{$\begin{array}{l}\text { Diabetic } \\
\text { retinopathy }\end{array}$} & \multirow{2}{*}{$\begin{array}{c}\text { Acute } \\
\text { kidney } \\
\text { Injury }\end{array}$} & \multirow{2}{*}{$\begin{array}{l}\text { Any } \\
\text { AE, } n \\
(\%)\end{array}$} & \multirow{2}{*}{$\begin{array}{c}\text { Gastrointestinal, } \\
\%\end{array}$} \\
\hline & & Nausea & Vomiting & Diarrhea & Pancreatitis & $\begin{array}{l}\text { Pancreatic } \\
\text { cancer }\end{array}$ & & & & & & \\
\hline
\end{tabular}

\section{SUSTAIN JAPAN}

'SITA'

S.c. semaglutide $0.5 \mathrm{mg} \quad 77$

S.c. (74.8)

$\begin{array}{lc}\text { S.c. semaglutide } 1 \mathrm{mg} \quad & 73 \\ & (71.6)\end{array}$

Sitagliptin $100 \mathrm{mg}$

68

(66.0)

SUSTAIN JAPAN

'INDIVIDUAL'

S.c. semaglutide $0.5 \mathrm{mg} \quad 206$

$3(1.3)$

S.c. semaglutide $1 \mathrm{mg}$

$(86.2)$
212

$6(2.5)$

Additional OAD

$(88)$
86

(investigators discretion) (71.7)

SUSTAIN China

S.c. semaglutide $0.5 \mathrm{mg} \quad 209$

(72.8\%)

$(72.8 \%)$
216

Sitagliptin $100 \mathrm{mg}$

$(74,5 \%)$

$(68,6 \%)$

0
$1(1.0)$
0
$3(1.3)$
$6(2.5)$
$2(1.7)$
$2(0.7 \%)$
$6(2.1 \%)$
$4(1.4 \%)$

(10.7)

(6.8\%)

0

$01(1.0)$

$4(3.9)$

NR

$3(2.9)$

NR

(12.7)

(8.8\%)

(1.9\%)

0

$1(1.0)$

$3(2.9)$

$2(1.9)$

NR

11

$(10.8)$
$2(1.9)$

NR

0

4 (3.9)

NR

$\begin{array}{ccc}29 & 13(5.4) & 24(10.0) \\ (12.1) & & \\ 46 & 14(5.8) & 38(15.8) \\ (19.1) & & \\ 1(0.8) & 2(1.7) & 8(6.7)\end{array}$

0
0
0

0

$4(1.7 \%)$

$11(4.6)$

NR

$A E$, adverse event; $E R$, extended release; NR, not reported; $O A D$, oral antidiabetic drug; s.c. subcutaneous.

An independent external adjudication committee (EAC) validated prespecified categories of adverse events (including deaths, selected cardiovascular events, malignant neoplasms, thyroid diseases [malignant thyroid neoplasms and C-cell hyperplasia], acute kidney injury, acute pancreatitis, and lactic acidosis) except in SUSTAIN 10 where there was no adjudication.

*An episode that was severe according to the ADA classification (requires assistance of another person to actively administer carbohydrate, glucagon, or other corrective action) or an episode with confirmed blood glucose value <56 mg/ dLand symptoms consistent with hypoglycemia. 